\title{
Oncological and functional outcomes of robot- assisted radical cystectomy in bladder cancer patients in a single tertiary center: Can these be preserved throughout the learning curve?
}

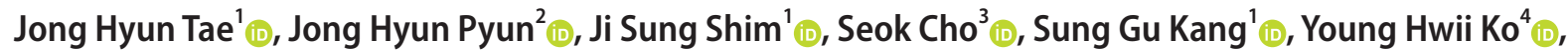 \\ Jun Cheon ${ }^{1}$ (D), Jeong Gu Lee ${ }^{1}$ (D), Seok Ho Kang ${ }^{1}$ (D) \\ 'Department of Urology, Korea University Medical Center, Korea University School of Medicine, Seoul, '² Department of Urology, Kangbuk Samsung Hospital, Seoul, \\ ${ }^{3}$ Department of Urology, Inje University Ilsan Paik Hospital, Inje University College of Medicine, Goyang, ${ }^{4}$ Department of Urology, Yeungnam University College of \\ Medicine, Daegu, Korea
}

Purpose: To evaluate the overall and segmental oncological and functional outcome of robot-assisted radical cystectomy (RARC) during the learning curve.

Materials and Methods: From August 2007 to November 2017, a total of 120 bladder cancer patients were treated with RARC in a single-tertiary hospital. These were divided into three groups of 40 consecutive cases. Overall and subgroup analysis of each group was used to evaluate oncological and functional outcomes throughout the learning curve.

Results: Among the 120 RARC cases, 42, 73, and 5 patients received extracorporeal urinary diversion (ECUD), intracorporeal urinary diversion (ICUD), and ureterocutaneostomy, respectively. There was a transition from ECUD to ICUD during the learning curve. The positive surgical margin rate was $0.8 \%$. The mean lymph node yield for the standard and extended pelvic lymph node dissection was 12.5 and 30.1, respectively, and increased to 19.8 and 31.2 and further to 20.0 and 37.9 , respectively, with each additional series of 40 cases. The 5-year overall survival and 3-year recurrence-free survival rates were $86.6 \%$ and $81.4 \%$, respectively. The 1-year daytime continence rate was $75.7 \%$, while the nighttime continence rate was $51.4 \%$. The potency preservation rate was $66.7 \%(n=8)$ with or without phosphodiesterase-5 inhibitors (PDE5-I) at 1 year and 33.3\% without PDE5-I ( $n=4)$.

Conclusions: RARC results in comparable oncological and functional outcomes to open radical cystectomy. In addition, the oncological and functional outcomes were well maintained throughout the learning curve. ECUD transition to ICUD was safe and did not compromise oncological or functional outcome.

Keywords: Cystectomy; Robotics; Urinary bladder neoplasms

This is an Open Access article distributed under the terms of the Creative Commons Attribution Non-Commercial License (http://creativecommons.org/licenses/by-nc/4.0) which permits unrestricted non-commercial use, distribution, and reproduction in any medium, provided the original work is properly cited.

Received: 9 April, 2019 • Accepted: 4 August, 2019

Corresponding Author: Seok Ho Kang (iD https://orcid.org/0000-0002-1524-5233

Department of Urology, Korea University Anam Hospital, 73 Goryeodae-ro, Seongbuk-gu, Seoul 02841, Korea

TEL: +82-2-920-5534, FAX: +82-2-928-7864, E-mail: mdksh@korea.ac.kr 


\section{INTRODUCTION}

Radical cystectomy with pelvic lymph node dissection (PLND) is currently the gold standard treatment for muscleinvasive bladder cancer and high-risk recurrent bladder cancer [1]. However, the procedure is associated with high perioperative morbidity [2]. Recent technological advancements in the field of minimally invasive surgery have led to novel techniques such as laparoscopic radical cystectomy and robot-assisted radical cystectomy (RARC) in an effort to reduce the high perioperative morbidity associated with open radical cystectomy (ORC) [3]. The potential benefits include decreased estimated blood loss, fewer transfusions, enhanced recovery of bowel function, shorter length of stay, and fewer postoperative complications $[4,5]$. Since the first publication in 2003 describing its use, the application of RARC has gradually increased with increasing evidence revealing its feasibility as an alternative to open surgery in bladder can- cer treatment [6,7]. In the United States, RARC has gradually increased from $16.7 \%$ in 2010 to $25.3 \%$ in 2013 , while ORC procedures have declined from $73.7 \%$ to $60.6 \%$ over the same period $[8,9]$. However, complication rates reported for RARC with extracorporeal urinary diversion (ECUD) remain substantial which has lead current trends to move beyond ECUD towards RARC with totally intracorporeal urinary diversion (ICUD) as a means for achieving better postoperative outcomes [5,10-12]. Such notions are due to the possibility of reduced gastrointestinal complication rates associated with less bowel manipulation and mobilization during ICUD procedures and limited peritoneal cavity exposure to air [13] However, major concerns are associated with the transition from ECUD to ICUD because of the steep learning curve and the ambiguity of impact on oncological and functional outcome. The primary endpoint of this study was to evaluate RARC in terms of its transition from ECUD to ICUD and to investigate its oncological and functional outcome.
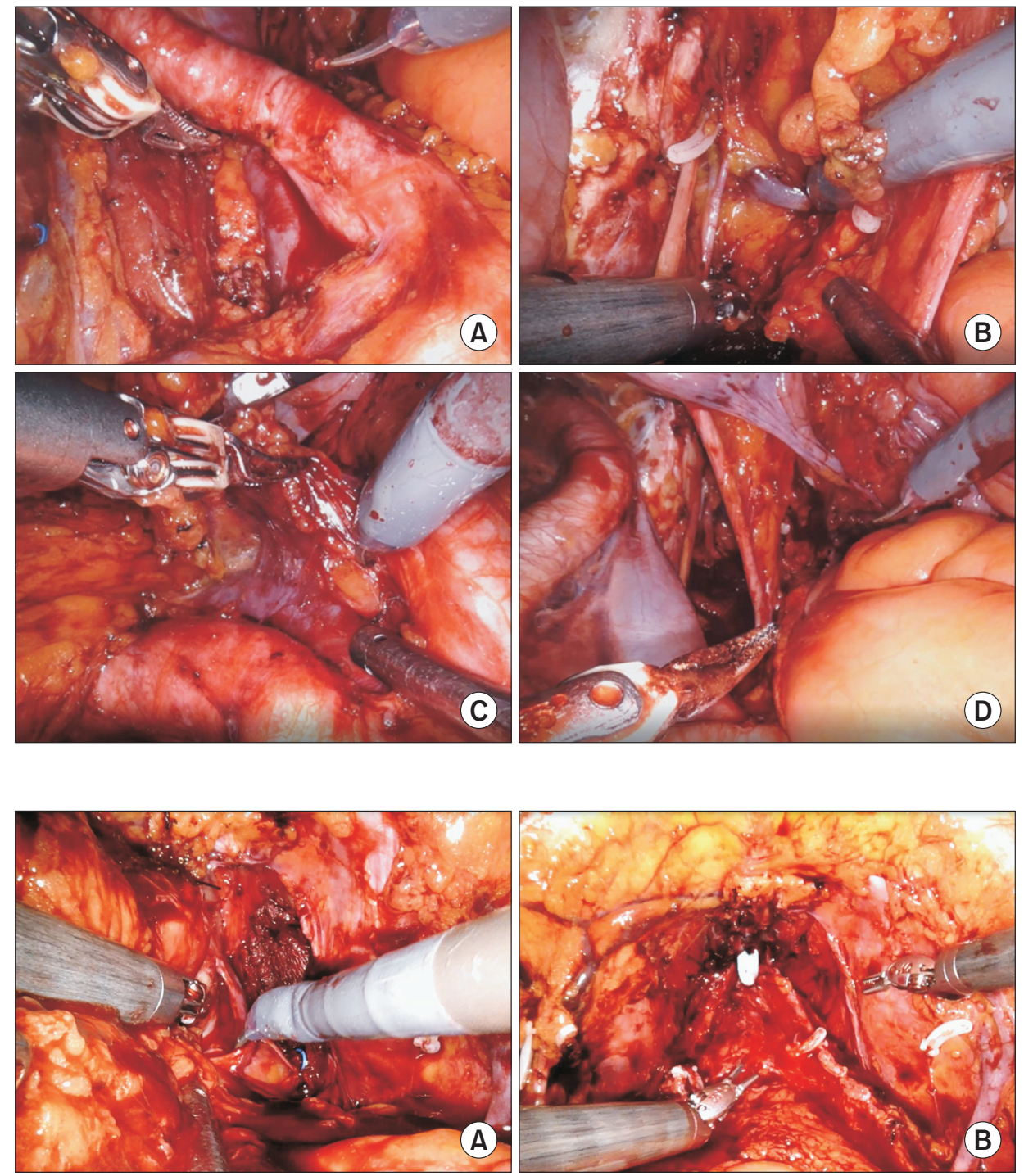

Fig. 1. Extended pelvic lymphadenectomy. (A) Triangle of Marcille. (B) Deep obturator. (C) Aortic bifurcation. (D) Left side final aspect.

Fig. 2. Neurovascular bundle preservation. (A) Intrafascial dissection of the neurovascular bundle from the lateral prostatic surface, left side. (B) The final aspect.

https://doi.org/10.4111/icu.2019.60.6.463 


\section{MATERIALS AND METHODS}

\section{Patients and study design}

This retrospective study was done according to the Declaration of Helsinki and approved by the Korea University Institutional Review Board (IRB no. 2010AN0168). Informed consent for research was obtained from all patients prior to RARC. From August 2007 to November 2017, 120 bladder cancer patients were treated with RARC either with an ECUD, ICUD, or with a ureterocutaneostomy by a single surgeon at a tertiary referral hospital. All patients had proven invasive or recurrent high-grade urothelial carcinoma by transurethral resection of the bladder tumor. Data was collected from a retrospective database and divided into 3 subgroups in order of the operation date. Patient demographics such as sex, body mass index (BMI), perioperative conditions, and the location and type of urinary diversion were compared. Overall oncological and functional outcomes were derived from the 120 cases of RARC. Additional analyses of the 3 subgroups were performed to evaluate the oncological and functional outcomes across the learning curve.

Postoperative continence rates were investigated for 58 orthotopic neobladder cases with a minimum of 1 year of follow-up. Continence rates for post operation 1, 3, 6, and 12 months derived and checked to see if clean intermittent catheterization (CIC) was needed at any point throughout the follow-up period. Daytime and nighttime continence was defined as the usage of 0 to 1 safety pad.

Postoperative functional outcome for sexual potency was derived from a patient questionnaire survey, the International Index of Erectile Function (IIEF)-5, taken preoperatively and postoperatively at 3,6 , and 12 months. Forty patients with either an orthotopic neobladder substitution $(n=23)$ or ileal conduit $(n=17)$ satisfied a minimum followup period of 12 months and completed all questionnaires. These cases were analyzed to evaluate potency preservation.
Potency was defined as the ability to maintain rigidity satisfactory for penetration throughout intercourse on more than half of the attempts with or without the aid of phosphodiesterase-5 inhibitors (PDE5-I).

\section{Surgical technique}

All patients underwent RARC with either a standard or an extended pelvic lymphadenectomy (Fig. 1). A classical Studer reservoir was adopted for orthotopic neobladder substitution. The surgical techniques for RARC with PLND from basic patient positioning and port placement to the boundaries of standard and extended pelvic lymphadenectomy have been described previously [14], along with detailed descriptions of the ICUD and neurovascular bundle preservation techniques [15] (Fig. 2).

For radical cystectomies with orthotopic neobladder substitutions in female patients, standard female radical cystectomy with anterior pelvic exenteration was performed with additional round ligament suspension (RLS). The classical technique of anterior pelvic exenteration involving en bloc resection of the bladder, urethra, uterus, fallopian tubes, ovaries, and anterior vaginal wall together with lymphadenectomy is described in literature. For RLS, as described by Ali-El-Dein et al. [16], the round ligaments are cut close to the uterus and later anchored to the lateral ends of the vaginal stump for posterior support (Fig. 3A). In carefully selected patients, reproductive organ-sparing radical cystectomy (ROSRC) was performed rather than the classical technique of anterior pelvic exenteration (Fig. 3B, C). Indications for ROSRC were younger age, desire for preservation of sexual function and/or fertility, unifocal tumors, and organ-confined tumors $(\leq \mathrm{T} 2 \mathrm{~b})$ that had no involvement of the bladder neck, trigone, or bladder base [17].

\section{Statistical analysis}

Patient demographics, clinical and pathologic variables
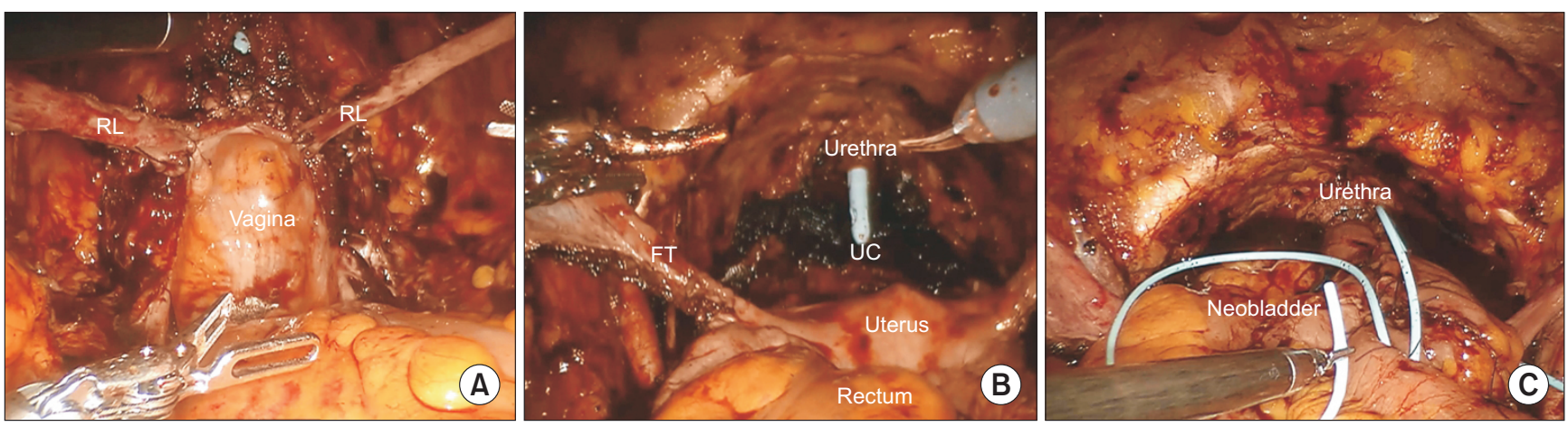

Fig. 3. Female robot-assisted radical cystectomy. (A) Round ligament suspension. (B) Reproductive organ-sparing radical cystectomy. (C) The final aspect. RL, round ligament; FT, fallopian tube; UC, urethral catheter. 
were analyzed using descriptive statistics. Fisher's exact test and chi-square test were used for categorical variables. Survival analysis was performed via Kaplan-Meier statistics and the log-rank test for comparisons. Analysis of covariance (ANCOVA) was used to derive the $p$-value for continuous variables across the learning curve. PASW Statistics version 18.0 (SPSS Inc., Chicago, IL, USA) was used for statistics, and a $p$-value $<0.05$ was considered significant.

\section{RESULTS}

\section{Patient demographics}

A summary of the patient demographics is presented in Table 1. There were no significant differences between the

Table 1. Demographics and characteristics of 120 robot-assisted radical cystectomy cases, overall and by patient series in chronological order of receipt of surgery

\begin{tabular}{|c|c|c|c|c|c|}
\hline \multirow{2}{*}{ Characteristic } & \multicolumn{5}{|c|}{ Case series } \\
\hline & $1-40$ & $41-80$ & $81-120$ & Total & $p$-value \\
\hline Number of patients & 40 & 40 & 40 & 120 & \\
\hline Age $(y)$ & $63.3 \pm 9.8$ & $65.7 \pm 11.2$ & $64.4 \pm 11.6$ & $64.5 \pm 11.0$ & $0.628^{\mathrm{a}}$ \\
\hline Sex & & & & & $0.095^{b}$ \\
\hline Male & $36(90.0)$ & $36(90.0)$ & $30(75.0)$ & $102(85.0)$ & \\
\hline Female & $4(10.0)$ & $4(10.0)$ & $10(25.0)$ & $18(15.0)$ & \\
\hline Body mass index $\left(\mathrm{kg} / \mathrm{m}^{2}\right)$ & $24.8 \pm 2.9$ & $24.7 \pm 3.0$ & $23.7 \pm 3.6$ & $24.4 \pm 3.2$ & $0.325^{\mathrm{a}}$ \\
\hline \multicolumn{6}{|l|}{ Perioperative condition } \\
\hline Neoadjuvant chemotherapy & $5(12.5)$ & $7(17.5)$ & $2(5.0)$ & $14(11.7)$ & $0.215^{c}$ \\
\hline Adjuvant chemotherapy & $0(0.0)$ & $4(10.0)$ & $8(20.0)$ & $12(10.0)$ & $0.050^{c}$ \\
\hline ECUD cases & $34(85.0)$ & $7(17.5)$ & $1(2.5)$ & $42(35.0)$ & $0.000^{c}$ \\
\hline ICUD cases & $3(7.5)$ & $31(77.5)$ & $39(97.5)$ & $73(60.8)$ & $0.000^{c}$ \\
\hline Other & $3(7.5)^{d}$ & $2(5.0)^{d}$ & $0(0.0)$ & $5(4.2)$ & \\
\hline Type of urinary diversion & & & & & $0.004^{c}$ \\
\hline Ileal conduit & $22(55.0)$ & $24(60.0)$ & $11(27.5)$ & $57(47.5)$ & \\
\hline Orthotopic neobladder & $15(37.5)$ & $14(35.0)$ & $29(72.5)$ & $58(48.3)$ & \\
\hline Ureterocutaneostomy & $3(7.5)$ & $2(5.0)$ & $0(0.0)$ & $5(4.2)$ & \\
\hline
\end{tabular}

Values are presented as number only, mean \pm standard deviation, or number (\%).

ECUD, extracorporeal urinary diversion; ICUD, intracorporeal urinary diversion.

${ }^{\mathrm{a}}$ :Analysis of covariance, ${ }^{\mathrm{b}}$ :chi-square test, ${ }^{\mathrm{c}}$ :Fisher's exact test, ${ }^{\mathrm{d}}:$ other refers to ureterocutaneostomy.

Table 2. Oncological outcomes, overall and by patient series in chronological order of receipt of surgery

\begin{tabular}{|c|c|c|c|c|c|}
\hline Oncological result & $1-40$ & $41-80$ & $81-120$ & Total $(n=120)$ & $p$-value \\
\hline Pathologic T stage, N0 & & & & & $0.301^{\mathrm{a}}$ \\
\hline $\mathrm{T} 2$ or less & $30(75.0)$ & $26(65.0)$ & $20(50.0)$ & $76(63.3)$ & \\
\hline $\mathrm{T} 3$ or $\mathrm{T} 4$ & $6(15.0)$ & $4(10.0)$ & $8(20.0)$ & $18(15.0)$ & \\
\hline LN positive & $4(10.0)$ & $10(25.0)$ & $12(30.0)$ & $26(21.7)$ & $0.525^{\mathrm{a}}$ \\
\hline T any, N1 & $1(2.5)$ & $5(12.5)$ & $5(12.5)$ & $11(9.2)$ & \\
\hline T any, N2 & $3(7.5)$ & $4(10.0)$ & $4(10.0)$ & $11(9.2)$ & \\
\hline T any, N3 & $0(0.0)$ & $1(2.5)$ & $3(7.5)$ & $4(3.3)$ & \\
\hline Positive margin & $0(0.0)$ & $0(0.0)$ & $1(2.5)^{b}$ & $1(0.8)^{b}$ & \\
\hline Type of LND & & & & & $0.002^{\mathrm{a}}$ \\
\hline Standard & $17(42.5)$ & $4(10.0)$ & $7(17.5)$ & $28(23.3)$ & \\
\hline Extended & $23(57.5)$ & $36(90.0)$ & $33(82.5)$ & $92(76.7)$ & \\
\hline $\mathrm{LN}$ yield & $22.6 \pm 13.0$ & $30.0 \pm 13.2$ & $34.4 \pm 18.0$ & $29.0 \pm 15.6$ & $0.002^{c}$ \\
\hline Standard & $12.5 \pm 6.7$ & $19.8 \pm 8.5$ & $20.0 \pm 9.4$ & $15.4 \pm 8.2$ & $0.060^{c}$ \\
\hline Extended & $30.1 \pm 11.5$ & $31.2 \pm 13.2$ & $37.9 \pm 18.0$ & $33.1 \pm 14.9$ & $0.115^{c}$ \\
\hline
\end{tabular}

Values are presented as number (\%) or mean \pm standard deviation.

LN, lymph node; LND, lymph node dissection.

${ }^{a}$ :Chi-square test, ${ }^{b}$ :the final pathology report for the margin positive case was pT3aN0, ${ }^{c}$ :analysis of covariance. 
mean age (63.3 years vs. 65.7 years vs. 64.4 years), sex distribution (male: $90.0 \%$ vs. $90.0 \%$ vs. $75.0 \%)$, BMI $\left(24.8 \mathrm{~kg} / \mathrm{m}^{2}\right.$ vs. $24.7 \mathrm{~kg} / \mathrm{m}^{2}$ vs. $23.7 \mathrm{~kg} / \mathrm{m}^{2}$ ), and perioperative conditions such as history of neoadjuvant chemotherapy (12.5\% vs. $17.5 \%$ vs. $5.0 \%$ ) or adjuvant chemotherapy ( $0.0 \%$ vs. $10.0 \%$ vs. $20.0 \%)$ among the 3 subgroups; cases 1 to 40,41 to 80 , and 81 to 120 , respectively (Table 1 ).

However, there was a significant difference in location (intracorporeal vs. extracorporeal) and type of urinary diversion (ileal conduit vs. orthotopic neobladder vs. ureterocutaneostomy). As our series of RARC progressed, the number of ICUDs and orthotopic neobladders increased from an initial $7.5 \%$ to $97.5 \%$ and $37.5 \%$ to $72.5 \%$, respectively.

Overall, ECUD, ICUD, and ureterocutaneostomy were performed in 42,73 , and 5 patients, respectively. Within the ICUD group, 30 (41.1\%) and 43 (58.9\%) patients each received either an ileal conduit or orthotopic neobladder substitution, while in the ECUD group, 27 (64.3\%) and 15 (35.7\%) patients each received an ileal conduit and an orthotopic neobladder substitution, respectively.

\section{Oncological outcomes}

Oncological outcomes are summarized in Table 2. Of the 120 patients, 96 patients had a minimum follow-up of one year and were analyzed to determine the overall oncological outcome of RARC. The number of patients in pathologic stage $\leq \mathrm{T} 2$, pT3-4, and pTanyN1-3 were 64,14 , and 18, respectively. Also, the number of recurrences for each group were 9 (14.1\%, mean time of recurrence at 22.5 months), 1 (7.1\%, at 23 months), and 8 patients (44.4\%, at 9.5 months), respectively. Tumor recurrences were observed in 18 patients (18.8\%) with a mean time to recurrence of 17.8 months in which 14 patients $(77.8 \%)$ recurred within two years and 16 patients (88.9\%) within 3 years.

The extent of lymph node (LN) dissection gradually evolved towards a more challenging extended PLND during the learning curve and consequently, there was a significant increase in total $\mathrm{LN}$ yield from 22.6 to 30.0 and eventually to 34.4 every 40 cases $(p<0.05)$. For each type of $\mathrm{LND}$, there were no significant differences in nodal yields during the

Table 3. The two-year, three-year, and five-year survival outcomes in 96 patients with a minimum 1-year follow-up period

\begin{tabular}{cccc}
\hline Overall survival outcomes & OS (\%) & RFS (\%) & CSS (\%) \\
\hline 2 years & 95.3 & 84.4 & 96.3 \\
3 years & 89.4 & 81.4 & 93.3 \\
5 years & 86.6 & 78.7 & 93.3 \\
\hline
\end{tabular}

OS, overall survival; RFS, recurrence-free survival; CSS, cancer-specific survival. learning curve. There was 1 case (0.8\%) of positive soft tissue margin in which the final pathology report was pT3aNO.

The overall survival (OS), recurrence-free survival (RFS) and cancer-specific survival (CSS) were 95.3\%, 84.4\%, 96.3\% at 2 years; $89.4 \%, 81.4 \%, 93.3 \%$ at 3 years; and $86.6 \%, 78.7 \%, 93.3 \%$ at 5 years, respectively (Table 3). The 3-year OS according to pathologic stage is summarized in Table 4. OS, RFS, and CSS, for T2 or less, T3/T4, pTanyN1-3 were 92.6\%, 88.1\%, 94.5\% and $85.7 \%, 88.9 \%, 100 \%$ and $77.9 \%, 46.0 \%$, and $82.5 \%$, respectively.

There were no statistically significant differences in the two-year OS, RFS, CSS between segments of the learning curve (OS: $97.3 \%$ vs. $92.1 \%$ vs. 89.9 , p $>0.05$; RFS: $84.9 \%$ vs. $79.5 \%$ vs. $81.9 \%$, $p>0.05$; CSS: $97.3 \%$ vs. $92.1 \%$ vs. $94.6 \%$, $p>0.05$ ) (Table 5).

Peritoneal carcinomatosis was observed in 2 cases (2.2\%); both cases involved ICUD with orthotopic neobladder substitutions. The final pathology results in both cases were associated with high stage (pT3aN1).

\section{Functional outcomes (urinary continence)}

Functional outcomes for urinary continence are shown in Table 5. Among the 120 cases of RARC, orthotopic neobladder substitution was performed in 58 cases comprising 6 female and 52 male patients. Of the 52 male patients, 37 satisfied a minimum follow-up period of 1 year and were analyzed to determine the functional outcome for urinary continence. Daytime and nighttime continence rates at postoperative $1,3,6$, and 12 months were $32.4 \%, 62.2 \%, 67.6 \%$, $75.7 \%$, and $18.9 \%$, $35.1 \%, 40.5 \%$, and $51.4 \%$, respectively. Day-

Table 4. The 3-year OS, RFS, CSS in 96 patients with minimum 1-year follow-up according to pathology stage (median follow-up period of 47.1 months)

\begin{tabular}{cccc}
\hline Final pathology stage & OS (\%) & RFS (\%) & CSS(\%) \\
\hline T2 or Less, N0 $(n=64)$ & 92.6 & 88.1 & 94.5 \\
(T0/Tis/Ta/T1/T2) & & & \\
T3/T4, N0 (n=14) & 85.7 & 88.9 & 100 \\
T any, N 1, 2, 3 $(n=18)$ & 77.9 & 46.0 & 82.5 \\
\hline
\end{tabular}

OS, overall survival; RFS, recurrence-free survival; CSS, cancer-specific survival.

Table 5. The 2-year OS, RFS, CSS, overall and by patient series in chronological order of receipt of surgery

\begin{tabular}{lcccc}
\hline 2-year & $\mathbf{1 - 4 0 ( \% )}$ & $\mathbf{4 1 - 8 0 ( \% )}$ & $\mathbf{8 1 - 1 2 0}(\%)$ & $\begin{array}{c}\text { Total } \\
(\mathbf{n}=120)\end{array}$ \\
\hline OS & 97.3 & 92.1 & 89.9 & 95.3 \\
RFS & 84.9 & 79.5 & 81.9 & 84.4 \\
CSS & 97.3 & 92.1 & 94.6 & 96.3 \\
\hline
\end{tabular}

OS, overall survival; RFS, recurrence-free survival; CSS, cancer-specific survival. 
time continence rate at 1 year that remained incontinent at night was $29.7 \%$.

Continence rates for each consecutive group of 40 cases are summarized in Table 6. The daytime continence rates at 1 year for cases 1 to 40, 41 to 80, 81 to 120 were each $73.3 \%$, $76.9 \%, 77.8 \%$ while nighttime continence rates were $46.7 \%$, $46.2 \%$, and $66.7 \%$, respectively. There were no significant differences in daytime and nighttime continence rates between groups. The CIC rate decreased significantly from $33.3 \%$ to $15.4 \%$ and $0.0 \%$ eventually with every 40 cases. All cases of male CIC were due to neovesicourethral anastomotic stricture except for 1 case of dysfunctional voiding.

Basic characteristics and functional results for female orthotopic neobladders are summarized in Table 7. Among the 6 female orthotopic neobladder substitutions, 3 underwent a ROSRC while the rest had a classical anterior exenteration with RLS. Daytime and nighttime continence was gained within 1 year in all cases except for 1 case of RLS that showed stress urinary incontinence. One case of dysfunctional voiding requiring CIC occurred in the ROSRC group.

\section{Functional outcomes (potency recovery)}

Of the 120 RARC patients, 40 male RARC patients with either an orthotopic neobladder substitution or ileal conduit satisfied a minimum follow-up of one year. Indications for neurovascular bundle (NVB) preservation included preoperatively potent men with an IIEF-5 score of $>12$, clinical stage T2 or less without involvement of the bladder neck, and absence of neurological and penile diseases. Fifteen patients (34.1\%) satisfied these indications. However, 3 of these patients were suspected of advanced stage intra-operatively and were excluded from NVB preservation. Eventually, 12 patients underwent either a bilateral or unilateral NVB preservation (11 bilateral \& 1 unilateral). The average preoperative and postoperative IIEF-5 score for these 12 patients were 16.8 and 7.7 at one year. The potency preservation rate was $66.7 \%(n=8)$ with or without PDE5-I and $33.3 \%$ without

Table 6. Functional outcomes (urinary incontinence) in male patients $(n=37)$, overall and in patient series according to chronological order of receipt of surgery

\begin{tabular}{|c|c|c|c|c|c|}
\hline & $1-40$ & $41-80$ & $81-120$ & Total $(n=120)$ & $p$-value \\
\hline Neobladder (no. of cases) & 15 & 13 & 9 & 37 & \\
\hline \multicolumn{6}{|l|}{ Daytime continence } \\
\hline At 1 month & $5(33.3)$ & $3(23.1)$ & $4(44.4)$ & $12(32.4)$ & $0.164^{\mathrm{a}}$ \\
\hline At 3 months & $8(53.3)$ & $9(69.2)$ & $6(66.7)$ & $23(62.2)$ & $0.653^{\mathrm{a}}$ \\
\hline At 6 months & $8(53.3)$ & $10(76.9)$ & $7(77.8)$ & $25(67.6)$ & $0.431^{\mathrm{a}}$ \\
\hline At 12 months & $11(73.3)$ & $10(76.9)$ & $7(77.8)$ & $28(75.7)$ & $0.211^{\mathrm{a}}$ \\
\hline \multicolumn{6}{|l|}{ Nighttime continence } \\
\hline At 1 month & $2(13.3)$ & $2(15.4)$ & $3(33.3)$ & $7(18.9)$ & $0.111^{\mathrm{a}}$ \\
\hline At 3 months & $4(26.7)$ & $4(30.8)$ & $5(55.6)$ & $13(35.1)$ & $0.373^{\mathrm{a}}$ \\
\hline At 6 months & $5(33.3)$ & $5(38.5)$ & $5(55.6)$ & $15(40.5)$ & $0.631^{\mathrm{b}}$ \\
\hline At 12 months & $7(46.7)$ & $6(46.2)$ & $6(66.7)$ & $19(51.4)$ & $0.644^{\mathrm{a}}$ \\
\hline Clean intermittent catheterization rate & $5(33.3)$ & $2(15.4)$ & $0(0.0)$ & $7(18.9)$ & $0.038^{\mathrm{a}}$ \\
\hline Vesico-urethral anastomosis site stricture rate & $5(33.3)$ & $1(7.7)$ & $0(0.0)$ & $6(16.2)$ & $0.031^{\mathrm{a}}$ \\
\hline
\end{tabular}

Values are presented as number only or number (\%).

${ }^{a}$ :Fisher's exact test, ${ }^{b}$ :chi-square test.

Table 7. Functional outcome (urinary incontinence) in female patients ( $n=6)$

\begin{tabular}{|c|c|c|c|c|c|c|}
\hline & Age & BMI $\left(\mathrm{kg} / \mathrm{m}^{2}\right)$ & Stage & $\begin{array}{c}\text { Daytime } \\
\text { continence gain (POD) }\end{array}$ & $\begin{array}{c}\text { Nighttime } \\
\text { continence gain (POD) }\end{array}$ & $\begin{array}{c}\text { Chronic } \\
\text { retention }(\mathrm{CIC})\end{array}$ \\
\hline 1 (ROSRC) & 55 & 20.6 & pT3aN0 & 6 months & 6 months & $x$ \\
\hline 2 (ROSRC) & 70 & 29.9 & pTisN0 & 2 months & 2 months & $\triangle$ \\
\hline $3(\mathrm{RLS})$ & 55 & 21.3 & pT3aN1 & Incontinence at 6 months & Incontinence at 6 months & $x$ \\
\hline 4 (RLS) & 61 & 22.1 & pT1N1 & 12 months & 12 months & $x$ \\
\hline 5 (RLS) & 57 & 18.3 & pT1N0 & 12 months & Incontinence at 1 year & $x$ \\
\hline 6 (ROSRC) & 58 & 25.2 & pT1N0 & 6 months & 6 months & $x$ \\
\hline
\end{tabular}

BMI, body mass index; POD, post-operative days; CIC, clean intermittent catheterization; ROSRC, reproductive organ-sparing radical cystectomy; $\mathrm{RLS}$, anterior exenteration and round ligament suspension; $\triangle$, combined voiding (voiding with partial $\mathrm{CIC}$ aid). 
PDE5-I $(n=4)$ at 1 year.

\section{DISCUSSION}

Acknowledged surrogate markers for oncological control and surgical quality include positive surgical margin (PSM) rates and LN yields. Comparing our results with historical data, Herr et al. [18] proposed optimal overall PSM rate (PSMR) $\leq 10 \%$ with a PSMR $\leq 15 \%$ in patients with T3-T4 bladder tumors, and the minimum number of LNs removed should be 10-14. The Pasadena Consensus Panel proposed that acceptable PSMR be $<7 \%$ overall, suggesting rates of $<3 \%$ for $\mathrm{pT} 2,<10 \%$ for $\mathrm{pT} 3$, and $<25 \%$ for pT4 [19]. PSMR reported for ORC in the RAZOR trial and Memorial Sloan Kettering Cancer Center (MSKCC) trial were 4.5\% and 5.2\%, respectively. The LN yield during LND in RARC reported in a systematic review by Yuh et al. [20] was 19 (range: 3-55), with half being an extended LND (yield range: 11-55). In our series, the PSMR was $0.8 \%$ overall and $5.6 \%$ for stage pT3NO. There were no PSMs in the pT2 or pTanyN1-3 groups. The mean total $\mathrm{LN}$ yield was 29.0. Therefore, the results of surrogate markers for our 120 cases of RARC are in line with historical data for ORC and currently available RARC results. In addition, these oncological surrogate markers are well established from the beginning and are maintained throughout the learning curve.

Long-term cancer-related deaths and RFS rates are the primary measurement of treatment efficacy in radical cystectomy. Due to lack of long-term follow-up in our study, comparisons were made based on large historical retrospective ORC series. A long-term analysis of survival in 1,000 ORC patients by Hautmann et al. [21] reported a five-year OS of $65.5 \%$. According to a systematic review of RARC outcome, 5-year and 3-year estimates for OS, RFS, and CSS were $39 \%$ to $66 \%, 53 \%$ to $74 \%, 66 \%$ to $80 \%$ and $61 \%$ to $80 \%$, $67 \%$ to $76 \%, 68 \%$ to $83 \%$, respectively [20]. According to the RAZOR trial, the 2-year RFS for RARC and ORC was 72.3\% vs. $71.6 \%$ and the 2 -year OS $80.2 \%$ vs. $79.1 \%$, respectively [5,1012]. The intermediate term OS, RFS, and CSS of this study seemed to present a comparable result to the ORC series and to currently available RARC results. Analysis of the learning curve indicated that the 2-year OS, RFS, CSS for cases 1 to 40,41 to 80,81 to 120 were $97.3 \%, 84.9 \%, 97.3 \%$ and $92.1 \%$, $79.5 \%, 92.1 \%$ and $89.9 \%, 81.9 \%, 94.6 \%$, respectively. The oncological outcome was maintained throughout the learning curve and the transition from ECUD to ICUD could be safely achieved without compromising the oncological outcome.

There were no significant differences in continence rates for male RARC throughout the learning curve. Previous publications have reported daytime continence rates to be $85 \%-100 \%$ and nighttime continence rates to be $60 \%$ to $95 \%$ for ORC [22,23]. However, other reports have indicated a wide array of continence rates from as low as $18 \%$ to as high as $92 \%$ at 1-year [24,25]. The variability is caused by the vast array of differences in the definition of continence, making comparison of continence rates difficult. Sharing our definition of continence as usage of 0 to 1 safety pad, a recent publication of male ORC showed daytime continence rates at 3,6 , and 12 months to be $34 \%, 47 \%$, and $71 \%$, and nighttime continence rates to be $14 \%, 25 \%$, and $37 \%$, respectively [26]. The reported daytime continence rates for RARC with orthotopic neobladder substitution ranged from $73.3 \%$ to $88.0 \%$ during the daytime and $55.5 \%$ to $58.1 \%$ at nighttime $[27,28]$ In our study, daytime and nighttime continence rates at postoperative $1,3,6$, and 12 months were each $32.4 \%, 62.2 \%$, $67.6 \%, 75.7 \%$, and $18.9 \%, 35.1 \%, 40.5 \%, 51.4 \%$, respectively. An early daytime continence gain of $32.4 \%$ at 1 month was noticeable. A postoperative 1-year pad-free daytime continence rate of $75.7 \%$ and 1-year pad-free nighttime continence rate of $51.4 \%$ were achieved. Despite the limited data for comparison, these results are in accordance with historical ORC and contemporary RARC literature. The daytime and nighttime continence rates during the learning curve at one year for cases 1 to 40,41 to 80,81 to 120 were $73.3 \%, 76.9 \%, 77.8 \%$ and $46.7 \%, 46.2 \%, 66.7 \%$, respectively. There were no significant differences in daytime and nighttime continence rates, implying that satisfactory continence rates are achieved early on in the learning curve and maintained throughout the transition to ICUD.

The majority of cases in which CIC was required were associated with neovesicourethral anastomotic stricture. Seven of 52 (13.5\%) male RARC patients with orthotopic neobladder substitutions required CIC. Six of the seven (85.7\%) CIC cases were related to neovesicourethral anastomotic stricture while the other was due to dysfunctional voiding. Five of six (83.3\%) neovesicourethral anastomotic strictures belonged to the initial 40 cases. These were all ECUD cases and required an additional surgical intervention of internal urethrotomy for the anastomotic stricture. The remaining one case of neovesicourethral anastomotic stricture was an ICUD and belonged to the 41 to 80 group which did not require surgical intervention but only periodic dilatation. In our experience, it seems as though neovesicourethral anastomotic stricture rates are higher in ECUD cases, possibly due to tensions created by gravity during a steep Trendelenburg position. Therefore, neovesicourethral anastomotic stricture may be prevented by minimizing tension during neovesicourethral anastomosis, resulting in a watertight seal with 
better blood supply and wound healing.

Potency recovery at one-year has been reported to range from $41 \%$ to $63 \%$ [29,30]. In our series of RARC, the potency recovery rate at one year was $66.7 \%$. These results are comparable to currently available results. Future prospective data collection and analysis to better evaluate the functional outcome is required.

Incontinence and hypercontinence rates following classical radical cystectomy with orthotopic neobladder substitutions have been reported to range from $34 \%$ to $44 \%$ and $35 \%$ to $61 \%$, respectively [17]. Hypercontinence has been described to be the result of downward and posterior migration of the neobladder during straining. Surgical modifications towards reducing chronic urinary retention rates include suspending the angles of the closed vaginal stump [16] In our series of female RARC, those not indicated for ROSRC underwent anterior exenteration with RLS. There were no cases of hypercontinence needing permanent CIC. Our data suggest that anterior superior fixation with RLS is advantageous in terms of preventing hypercontinence.

Potential limitations to this study include its retrospective, single-institution, nonrandomized study design with the possibility of selection bias for patients with lower tumor burden the early stages of RARC. Moreover, the short clinical and oncological follow-up period remain as potential limitations. Also, rather than a direct comparative analysis between ORC and RARC, we used historical ORC data as reference for comparison with RARC. Nevertheless, this study holds meaning in that both oncological and functional data for RARC are reported while current literature remain immature.

\section{CONCLUSIONS}

RARC results in comparable functional and oncological outcomes as in historical ORC series. Therefore, when oncological principals regarding ORC are followed, RARC can be an appropriate minimally invasive surgical option for patients with invasive bladder cancer. In addition, the oncological and functional outcomes are well maintained throughout the learning curve. Furthermore, the transition from ECUD to ICUD may be successfully achieved without compromising oncological and functional outcomes.

\section{CONFLICTS OF INTEREST}

The authors have no nothing to disclose.

\section{ACKNOWLEDGMENTS}

This research was supported by a grant of Korea University Medical Center and Anam Hospital, Seoul, Korea.

\section{REFERENCES}

1. Shariat SF, Karakiewicz PI, Palapattu GS, Lotan Y, Rogers CG, Amiel GE, et al. Outcomes of radical cystectomy for transitional cell carcinoma of the bladder: a contemporary series from the Bladder Cancer Research Consortium. J Urol 2006;176:2414-22; discussion 2422.

2. Challacombe BJ, Bochner BH, Dasgupta P, Gill I, Guru K, Herr $\mathrm{H}$, et al. The role of laparoscopic and robotic cystectomy in the management of muscle-invasive bladder cancer with special emphasis on cancer control and complications. Eur Urol 2011;60:767-75.

3. Guiote I, Gaya JM, Gausa L, Rodríguez O, Palou J. Complications from robot-assisted radical cystectomy: where do we stand? Actas Urol Esp 2016;40:108-14.

4. Parekh DJ, Reis IM, Castle EP, Gonzalgo ML, Woods ME, Svatek RS, et al. Robot-assisted radical cystectomy versus open radical cystectomy in patients with bladder cancer (RAZOR): an open-label, randomised, phase 3, non-inferiority trial. Lancet 2018;391:2525-36.

5. Bochner BH, Dalbagni G, Sjoberg DD, Silberstein J, Keren Paz GE, Donat SM, et al. Comparing open radical cystectomy and robot-assisted laparoscopic radical cystectomy: a randomized clinical trial. Eur Urol 2015;67:1042-50.

6. Menon M, Hemal AK, Tewari A, Shrivastava A, Shoma AM, El-Tabey NA, et al. Nerve-sparing robot-assisted radical cystoprostatectomy and urinary diversion. BJU Int 2003;92:232-6.

7. Nix J, Smith A, Kurpad R, Nielsen ME, Wallen EM, Pruthi RS. Prospective randomized controlled trial of robotic versus open radical cystectomy for bladder cancer: perioperative and pathologic results. Eur Urol 2010;57:196-201.

8. Bachman AG, Parker AA, Shaw MD, Cross BW, Stratton KL, Cookson MS, et al. Minimally invasive versus open approach for cystectomy: trends in the utilization and demographic or clinical predictors using the National Cancer Database. Urology 2017;103:99-105.

9. Hanna N, Leow JJ, Sun M, Friedlander DF, Seisen T, Abdollah F, et al. Comparative effectiveness of robot-assisted vs. open radical cystectomy. Urol Oncol 2018;36:88.e1-9.

10. Parekh DJ, Messer J, Fitzgerald J, Ercole B, Svatek R. Perioperative outcomes and oncologic efficacy from a pilot prospective randomized clinical trial of open versus robotic assisted radical cystectomy. J Urol 2013;189:474-9.

11. Khan MS, Gan C, Ahmed K, Ismail AF, Watkins J, Summers 
JA, et al. A single-centre early phase randomised controlled three-arm trial of open, robotic, and laparoscopic radical cystectomy (CORAL). Eur Urol 2016;69:613-21.

12. Parekh D. Pnflba-18: a prospective, multicenter, randomized trial of open versus robotic radical cystectomy (RAZOR). J Urol 2017;197:e918.

13. Augestad KM, Delaney CP. Postoperative ileus: impact of pharmacological treatment, laparoscopic surgery and enhanced recovery pathways. World J Gastroenterol 2010;16:2067-74.

14. Kang SG, Kang SH, Lee YG, Rha KH, Jeong BC, Ko YH, et al. Robot-assisted radical cystectomy and pelvic lymph node dissection: a multi-institutional study from Korea. J Endourol 2010;24:1435-40.

15. Kang SG, Ko YH, Jang HA, Kim J, Kim SH, Cheon J, et al. Initial experience of robot-assisted radical cystectomy with total intracorporeal urinary diversion: comparison with extracorporeal method. J Laparoendosc Adv Surg Tech A 2012;22:456-62.

16. Ali-El-Dein B, Gomha M, Ghoneim MA. Critical evaluation of the problem of chronic urinary retention after orthotopic bladder substitution in women. J Urol 2002;168:587-92.

17. Niver BE, Daneshmand S, Satkunasivam R. Female reproductive organ-sparing radical cystectomy: contemporary indications, techniques and outcomes. Curr Opin Urol 2015;25:10510.

18. Herr H, Lee C, Chang S, Lerner S; Bladder Cancer Collaborative Group. Standardization of radical cystectomy and pelvic lymph node dissection for bladder cancer: a collaborative group report. J Urol 2004;171:1823-8; discussion 1827-8.

19. Wilson TG, Guru K, Rosen RC, Wiklund P, Annerstedt M, Bochner BH, et al.; Pasadena Consensus Panel. Best practices in robot-assisted radical cystectomy and urinary reconstruction: recommendations of the Pasadena Consensus Panel. Eur Urol 2015;67:363-75.

20. Yuh B, Wilson T, Bochner B, Chan K, Palou J, Stenzl A, et al. Systematic review and cumulative analysis of oncologic and functional outcomes after robot-assisted radical cystectomy. Eur Urol 2015;67:402-22.

21. Hautmann RE, de Petriconi RC, Volkmer BG. 25 years of experience with 1,000 neobladders: long-term complications. J Urol
2011;185:2207-12.

22. Lee RK, Abol-Enein H, Artibani W, Bochner B, Dalbagni G, Daneshmand S, et al. Urinary diversion after radical cystectomy for bladder cancer: options, patient selection, and outcomes. BJU Int 2014;113:11-23.

23. Hautmann RE, Abol-Enein H, Davidsson T, Gudjonsson $\mathrm{S}$, Hautmann SH, Holm HV, et al.; International Consultation on Urologic Disease-European Association of Urology Consultation on Bladder Cancer 2012. ICUD-EAU International Consultation on Bladder Cancer 2012: urinary diversion. Eur Urol 2013;63:67-80.

24. Novara G, Ficarra V, Minja A, De Marco V, Artibani W. Functional results following vescica ileale Padovana (VIP) neobladder: midterm follow-up analysis with validated questionnaires. Eur Urol 2010;57:1045-51.

25. Koie T, Hatakeyama S, Yoneyama T, Ishimura H, Yamato T, Ohyama C. Experience and functional outcome of modified ileal neobladder in 95 patients. Int J Urol 2006;13:1175-9.

26. Nayak AL, Cagiannos I, Lavallée LT, Morash C, Hickling D, Mallick R, et al. Urinary function following radical cystectomy and orthotopic neobladder urinary reconstruction. Can Urol Assoc J 2018;12:181-6.

27. Schwentner C, Sim A, Balbay MD, Todenhöfer T, Aufderklamm S, Halalsheh $\mathrm{O}$, et al. Robot-assisted radical cystectomy and intracorporeal neobladder formation: on the way to a standardized procedure. World J Surg Oncol 2015;13:3.

28. Simone G, Papalia R, Misuraca L, Tuderti G, Minisola F, Ferriero $\mathrm{M}$, et al. Robotic intracorporeal padua ileal bladder: surgical technique, perioperative, oncologic and functional outcomes. Eur Urol 2018;73:934-40.

29. Jonsson MN, Adding LC, Hosseini A, Schumacher MC, Volz D, Nilsson A, et al. Robot-assisted radical cystectomy with intracorporeal urinary diversion in patients with transitional cell carcinoma of the bladder. Eur Urol 2011;60:1066-73.

30. Tyritzis SI, Hosseini A, Collins J, Nyberg T, Jonsson MN, Laurin $\mathrm{O}$, et al. Oncologic, functional, and complications outcomes of robot-assisted radical cystectomy with totally intracorporeal neobladder diversion. Eur Urol 2013;64:734-41. 